Field-effect control of tunneling barrier height by exploiting graphene's low density of states

L. A. Ponomarenko, B. D. Belle, R. Jalil, L. Britnell, R. V. Gorbachev, A. K. Geim, K. S. Novoselov, A. H. Castro Neto, L. Eaves, and M. I. Katsnelson

Citation: Journal of Applied Physics 113, 136502 (2013); doi: 10.1063/1.4795542

View online: http://dx.doi.org/10.1063/1.4795542

View Table of Contents: http://aip.scitation.org/toc/jap/113/13

Published by the American Institute of Physics 


\title{
Field-effect control of tunneling barrier height by exploiting graphene's low density of states
}

\author{
L. A. Ponomarenko, ${ }^{1}$ B. D. Belle, ${ }^{2}$ R. Jalil, ${ }^{2}$ L. Britnell, ${ }^{1}$ R. V. Gorbachev ${ }^{2}$ A. K. Geim,,${ }^{1,2}$ \\ K. S. Novoselov, ${ }^{1}$ A. H. Castro Neto, ${ }^{3}$ L. Eaves, ${ }^{1,4}$ and M. I. Katsnelson ${ }^{5}$ \\ ${ }^{1}$ School of Physics \& Astronomy, University of Manchester, Manchester M13 9PL, United Kingdom \\ ${ }^{2}$ Manchester Centre for Mesoscience \& Nanotechnology, University of Manchester, Manchester M13 9PL, \\ United Kingdom \\ ${ }^{3}$ Graphene Research Center \& Department of Physics, National University of Singapore, Singapore 117542 \\ ${ }^{4}$ School of Physics \& Astronomy, University of Nottingham, Nottingham NG7 2RD, United Kingdom \\ ${ }^{5}$ Institute for Molecules and Materials, Radboud University of Nijmegen, 6525 AJ Nijmegen, The Netherlands
}

(Received 29 August 2012; accepted 22 January 2013; published online 29 March 2013)

\begin{abstract}
We exploit the low density of electronic states of graphene to modulate the tunnel current flowing perpendicular to the atomic layers of a multi-layer graphene-boron nitride device. This is achieved by using the electric field effect to raise the Fermi energy of the graphene emitter layer and thereby reduce the effective barrier height for tunneling electrons. We discuss how the electron charge density in the graphene layers and the properties of the boron nitride tunnel barrier determine the device characteristics under operating conditions and derive expressions for carrier tunneling in these highly anisotropic layered heterostructures. (C) 2013 American Institute of Physics.
\end{abstract}

[http://dx.doi.org/10.1063/1.4795542]

\section{INTRODUCTION}

In a recent article, we described the fabrication and properties of a bipolar field-effect tunnel transistor that exploits the low density of electronic states of single atomic layer crystalline graphene. ${ }^{1}$ The device is a multilayer heterostructure in which two graphene electrodes are sandwiched between a few atomic layers of hexagonal boron nitride (hBN) which acts as a high quality tunnel barrier. ${ }^{2}$ In tunnel diodes and transistors based on conventional semiconductor heterostructure materials, ${ }^{3-7}$ the tunnel current is tuned by the bias-induced voltage drop across the barrier which changes its potential height. In contrast, we exploit a unique feature of graphene, namely its low density of states near the Dirac point. By applying a gate voltage, we can induce a large increase in the Fermi energy of the graphene emitter layer. This so-called "quantum capacitance" effect ${ }^{8}$ is usually small in the case of carrier tunneling between two quantum wells (QWs) made from III-V heterostructures due to the finite effective mass of the charge carriers. The sheet density of carriers in the QW induced by an applied bias produces a change of Fermi energy which is small compared to the height of the tunnel barrier. In contrast, for graphene, the corresponding reduction of the effective barrier height significantly increases the tunneling transmission coefficient of the barrier.

Following a brief discussion of the device structure and its operation, we present a model to explain the currentvoltage characteristics, $I-V$, of our device by considering the charge distribution in the graphene layers and the band structure of the h-BN tunnel barrier. This is followed by a discussion of the reproducibility of the current-voltage characteristics over a series of samples and of the effects of variations in barrier thickness and by a conclusion and outlook for future work.

\section{DEVICE COMPOSITION AND CHARGE ON ELECTRODES UNDER BIAS}

The geometry of our device is shown schematically in Fig. 1. A thick layer of h-BN is placed on top of the oxidized surface of a doped Si substrate and acts as a high-quality, atomically-flat surface and as a lower encapsulation layer on which the active part of the device is mounted. This consists of two graphene electrodes on either side of an atomically thin h-BN tunnel barrier, whose thickness was determined by atomic force microscopy, Raman microscopy and optical contrast. ${ }^{9}$ The graphene electrodes are in the form of Hall bars to allow measurements of the electrical properties of each layer. The doped silicon substrate serves as a gate electrode, which provides additional control of the electrical properties of the two graphene layers.

A gate voltage, $V_{g}$, is applied between the Si-doped layer and the bottom graphene layer; $D$ is the separation between the edge of the doped Si layer and the bottom graphene electrode. When the Si-doped layer is biased positive, the electric field, $F_{g}=V_{g} / D$, in the oxide layer induces an increase in the electron density, and hence the Fermi energy, $E_{F}$, in the bottom graphene electrode. A tunnel current flows between the bottom $\left(\mathrm{Gr}_{\mathrm{B}}\right)$ and top $\left(\mathrm{Gr}_{\mathrm{T}}\right)$ graphene electrodes when a bias voltage $V_{b}$ is applied between these layers. The induced carrier densities in the graphene electrodes $n_{\mathrm{T}}$ and $n_{\mathrm{B}}$ are related to $F_{g}$ and the applied electric field in the barrier layer, $F_{b}$, in the following way:

$$
\begin{gathered}
\left|F_{b}\right|=n_{T} e / \varepsilon_{0} \varepsilon_{r}, \\
\left|F_{g}\right|=\left(n_{B}+n_{T}\right) e / \varepsilon_{0} \varepsilon_{r} .
\end{gathered}
$$

The ambipolar nature of the carriers in graphene is included in the signs of $n_{T}$ and $n_{B}$. Since the relative permittivities, $\varepsilon_{r}$, of $\mathrm{SiO}_{2}$ and h-BN are similar, we assume them to 

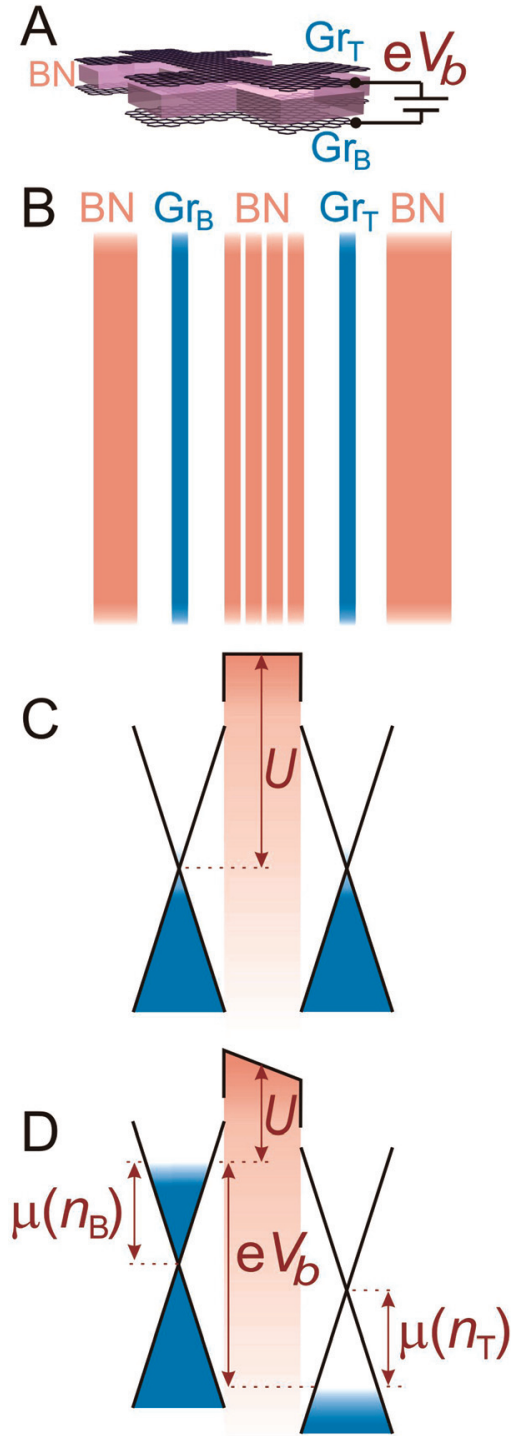

FIG. 1. (A) and (B) show the schematic diagram of the device structure. (C) and (D) show how the barrier height, $U$, for carriers at the emitter Fermi energy is strongly reduced due to the low density of states characteristic of graphene close to the neutrality point.

be equal, for simplicity. The bias voltage $V_{b}$ between the two graphene electrodes is then given by

$$
e V_{b}=e F_{b} d-\mu\left(n_{T}\right)+\mu\left(n_{B}\right),
$$

where $d$ is the h-BN thickness and $\mu(n)$ are the chemical potentials in the corresponding graphene layers. For simplicity, we also assume that graphene electrodes are chemically undoped so that $n_{\mathrm{T}}=n_{\mathrm{B}}=0$ in the absence of applied voltages.

Taking into account the electron-hole symmetry of graphene, $\mu(-n)=-\mu(n)$, we obtain the following relation:

$$
\frac{e^{2} d}{\varepsilon_{0} \varepsilon_{r}} n_{T}+\mu\left(n_{T}\right)+\mu\left(n_{T}+\frac{\varepsilon_{0} \varepsilon_{r} F_{g}}{e}\right)+e V_{b}=0 .
$$

This equation allows us to determine the sheet density, $n_{T}$, induced by the field effect in the top graphene layer, $G r_{T}$, for a given $V_{g}$. For a conventional two-dimensional (2D) gas with massive electrons, $\mu(n) \propto n$. In this case, the first term in eq. (1), which describes the classical capacitance of the tunnel barrier, dominates for any realistic values of $d$ that are larger than an interatomic distance. In contrast, for graphene, with its low density of states and Dirac-like spectrum, $\mu(n) \propto \sqrt{n}$. This leads to a qualitatively different behaviour, which can be described in terms of a quantum capacitance ${ }^{8,10}$ - see also Refs. 11 and 12 for a discussion of the effect of doping.

By using the above expressions to determine $n_{\mathrm{T}}$ and $n_{\mathrm{B}}$ as a function of bias $V_{b}$ and gate voltage $V_{g}$, we can model the measured I-V characteristics of the device reported in Fig. 3 of Ref. 1. To illustrate the good agreement between experiment and theory, Figure 2 shows the experimentally measured carrier concentrations in the top and bottom graphene layer $n\left(V_{g}\right)$ and compares them with the behavior expected from solving Eq. (1).

\section{MODELING THE CURRENT-VOLTAGE CHARACTERISTICS}

The $I-V$ curves for a tunnel junction are generally described by the relation

$I(V) \propto \int d E D o S_{B}(E) D o S_{T}(E-e V) T(E)[f(E-e V)-f(E)]$,

where $f(E)$ is the Fermi distribution function. At low temperatures, the difference between the Fermi functions restricts the relevant energy $E$ integral to $\mu<E<\mu+e V$ where $\mu$ is the chemical potential. To be specific, we consider the case $e V>0$. Relation (2) above assumes that in-plane momentum is not conserved in the tunneling process. This is a reasonable assumption for the case of graphene-h-BN interfaces, since there are several possible mechanisms for elastic scattering at the interfaces, for example, unavoidable fluctuations

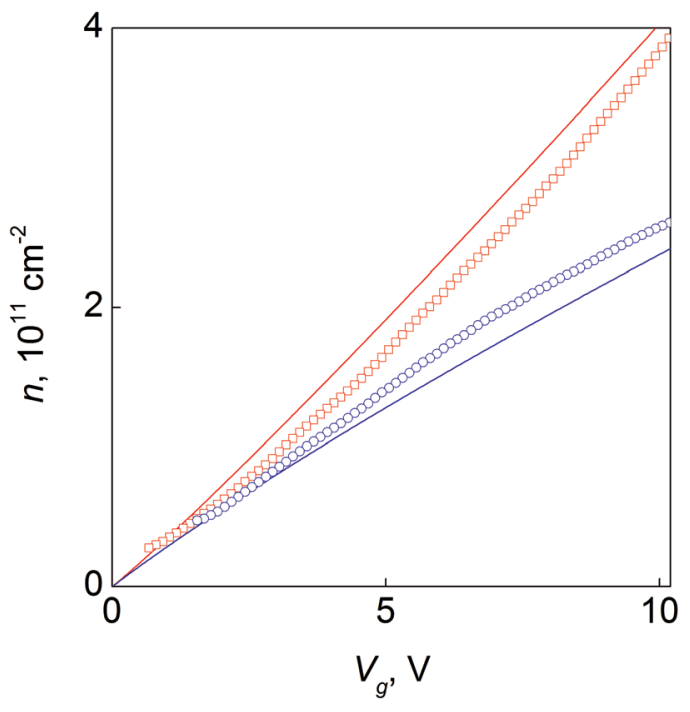

FIG. 2. Nonlinear dependence of charge carrier concentrations in the two graphene electrodes as a function of gate voltage. The symbols are experimental data (red symbols for the bottom graphene layer; blue for the top). The solid curves in the corresponding colours are our modelling. No fitting parameters are used. 
due to lattice mismatch. ${ }^{13}$ More importantly, this approach provides a reasonable fit to our measured $I-V$ characteristics.

If the tunneling conductance per channel is much smaller than the conductivity quantum $e^{2} / h$, as in our case, the transmission probability $T$ is exponentially small and depends strongly on the energy $E$ of tunneling electrons, as follows:

$$
T(E)=A(E) \exp [-W(E)]
$$

where $A$ is a smooth function that depends on the details of the wave-function matching at the interface. In our model, we assume that $A$ is a constant.

We now consider the functional form of $W(E)$. For the simple case of an isotropic barrier, we need to solve the dispersion equation $E=\varepsilon_{n}\left(k_{x}, k_{y}, k_{z}\right)$ for each band of the barrier material, where $E$ is the energy of isotropic electrons tunneling in the $z$ direction. There is no real solution for $k_{z}$ within the energy gap. The minimal imaginary part of $k_{z}$, $I m k_{z}$, for a given $E$ and arbitrary $k_{x}$ and $k_{y}$, which dominates the tunneling probability, is given by

$$
W(E)=2 d \operatorname{Im}_{z} .
$$

For the case of parabolic bands, $\operatorname{Im} k_{z}=\sqrt{2 m \Delta} / \hbar$ where $\Delta$ is the barrier height (in our case, the distance to the valence band) and $m$ is the effective mass. ${ }^{14-16}$

For a layered crystal, a better approximation to the band structure is

$$
\varepsilon\left(k_{x}, k_{y}, k_{z}\right)=\tau\left(k_{z}\right)+\varepsilon_{1}\left(k_{x}, k_{y}\right),
$$

where $\tau\left(k_{z}\right)=2 t_{\perp} \cos \left(k_{z} l\right) ; t_{\perp}$ describes the interlayer coupling and $l$ is the interlayer distance (for the case of h-BN, $l$ $\approx 3.4 \AA$ ). By solving the corresponding tunneling equation, we then find $k_{z}$ within the gap to be

$$
k_{z}=\frac{1}{l} \ln \left(\left|\frac{E-\varepsilon_{1}}{2 t_{\perp}}\right|+\sqrt{\left(\frac{E-\varepsilon_{1}}{2 t_{\perp}}\right)^{2}-1}\right) .
$$

The top of the valence band corresponds to $E_{\max }=\max \varepsilon_{1}$ $\left(k_{x}, k_{y}\right)+2 t_{\perp}$ (to be specific, we choose $t_{\perp}>0$ ), and the optimal value for the tunneling wave vector is then

$$
\operatorname{Im} k_{z}=\frac{1}{l} \ln \left(\left(\frac{\Delta}{2 t_{\perp}}+1\right)+\sqrt{\left(\frac{\Delta}{2 t_{\perp}}+1\right)^{2}-1}\right),
$$

where $\Delta=E-E_{\max }$. If $\Delta \gg 2 t_{\perp}$, this expression can be simplified to $k_{z}=i / l \ln \left(\Delta / t_{\perp}\right)$ and yields a tunnelling probability $T \propto\left(t_{\perp} / \Delta\right)^{2 \mathrm{n}}$ where $n=d / l$ is the number of atomic layers in the tunnel barrier. In the opposite limit, $\Delta \ll 2 t_{\perp}$, we obtain $k_{z}=(i / l) \sqrt{\Delta / t_{\perp}}=i \sqrt{2 m^{*} \Delta} / \hbar$ where $m^{*}=\hbar^{2} / 2 t_{\perp} l^{2}$ is the effective mass in the tunneling direction. This shows that the isotropic model is a reasonable approximation for layered crystals, provided that the tunneling occurs reasonably close to the band-gap edge.

Equation (4) is a simplified version of the real band structure of h-BN, which depends on stacking order. The layer stacking for h-BN crystals is usually of the $\mathrm{AA}^{\prime}$ type. ${ }^{17}$ An analytical solution can be obtained by neglecting the mixing of $\pi$ and $\sigma$ bands, ${ }^{18,19}$ giving the following dispersion relation:

$$
\varepsilon^{2}\left(k_{x}, k_{y}, k_{z}\right)=\frac{E_{g}^{2}}{4}+\tau^{2}\left(k_{z}\right)+\varepsilon_{1}^{2}\left(k_{x}, k_{y}\right) \pm 2 \tau\left(k_{z}\right) \varepsilon_{1}\left(k_{x}, k_{y}\right),
$$

where $E_{g}$ is the energy difference between boron and nitrogen sites. ${ }^{17}$ In this case, we find

$$
\operatorname{Im} k_{z}=\frac{1}{l} \ln \left(\frac{\Phi}{2 t_{\perp}}+\sqrt{\left(\frac{\Phi}{2 t_{\perp}}\right)^{2}-1}\right),
$$

where $\Phi=\sqrt{E^{2}-E_{g}^{2} / 4}-\left|\varepsilon_{1}\left(k_{x}, k_{y}\right)\right|$. Equation (7) differs from (5) by the substitution $E \rightarrow \sqrt{E^{2}-E_{g}^{2} / 4}$, which indicates the general validity of relation $\operatorname{Im} k_{z} \propto \ln (\Delta)$ for describing tunneling through strongly layered materials. Equations (5) and (7) fit our experimental data equally well. It is interesting to note that the tunnelling exponent through layered crystals depends on $E$ only weakly (logarithmically) in contrast to isotopic crystals which exhibit the conventional square-root energy dependence. For small changes in $\Delta$, this difference is unimportant (see below). Note also that in the case of a strong electric field such that it changes the rectangular shape of the tunnel barrier (see Fig. 1) the above expressions for $W$ can be generalized within the WKB approximation $^{16}$ as

$$
W=e \int_{0}^{d} d x \operatorname{Imk}_{z}(\Delta \rightarrow \Delta(x)) .
$$

\section{LAYERED VERSUS ISOTROPIC BARRIER}

In Ref. 1, we chose for the sake of brevity to ignore the fact that our tunnel barriers are composed of a strongly layered material. This simplification allowed us to apply a standard tunneling model. However, the assumption can be justified further by noting that, for our device parameters, there is no difference between the I-V characteristics calculated for the layered and isotropic materials; therefore, we cannot distinguish between the two cases. To illustrate this insensitivity to the layered structure of our tunnel barrier, Figure 3 shows experimental I-V curves for two devices and compares them with the behavior expected for layered and isotropic cases. No major difference can be seen, except at low bias in Fig. 3(a). The exact shape of experimental curves at low bias varies from sample to sample (cf. Figs. 3(a) and 3(b)) and, hence, we do not discuss this difference further.

A recent paper by Feenstra et al. ${ }^{12}$ has considered theoretically the single-particle tunneling of carriers between two graphene electrodes. They modeled the case when the two graphene sheets have unequal doping and assumed that the tunnel barrier was isotropic and neglected the effect of scattering in the barrier. Their results suggest that there should 

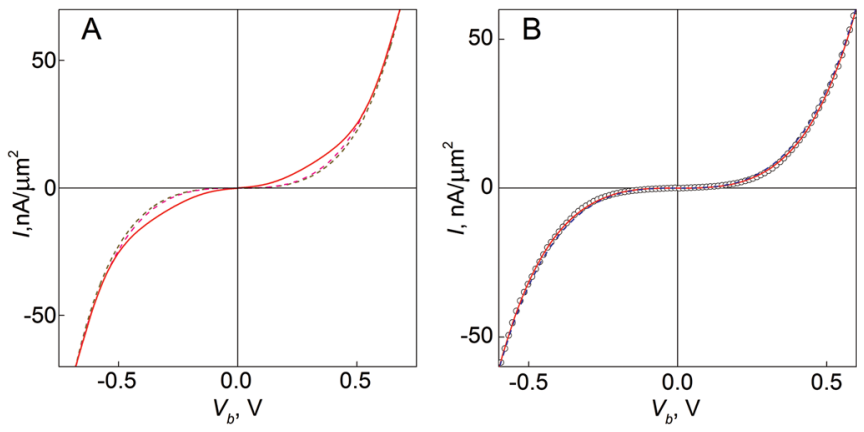

FIG. 3. Tunnelling I-V characteristics for two different devices, each with a 4-atomic layer h-BN barrier, at zero gate voltage and a comparison with theory. (A) The red solid curve is the experimental data. The two dashed curves are our modelling for an isotropic barrier ( $\Delta$ and $m$ as in Ref. 1) and for a layered barrier of the same height and $t_{\perp}=0.6 \mathrm{eV}$, by using the formulae given above. Note that $t_{\perp} \approx 0.6 \mathrm{eV}$ corresponds to $m=0.5 m_{0}$. (B) Nominally similar device (for clarity, the experimental data are shown by symbols). The curves are again the layered and isotropic versions of the tunnelling theory. The fitting parameter is the constant $A$ in Eq. (3), which determines the absolute value of the current. The close agreement between functional forms of the theoretical curves validates the use of the conventional tunnelling formulae in Ref. 1.

be a resonantly enhanced peak in the I-V characteristics of the device when the two graphene lattices are aligned. The effect of misalignment of the two graphene layers was also considered.

\section{EXAMPLES OF OUR DEVICE OPERATION}

We have studied 8 multi-terminal devices with Hall bar graphene electrodes (see Fig. 1) and more than 20 simpler tunneling FETs with only one or two Ohmic contacts attached to each graphene electrode. The latter structures do not provide as much information about the properties of the graphene electrodes, but even one contact is sufficient to study the tunneling I-V characteristics. Reference 1 reported the operation of such three-terminal graphene transistors (see Fig. 3 therein). To illustrate the degree of reproducibility for different samples, Figure 4 plots the behavior observed in another device with the tunnel barrier consisting of 4 layers of h-BN. One can see that the nonlinear I-V characteristics are qualitatively similar to those presented for the device described in detail in Ref. 1 , and their response to gate voltage is also similar.
The only consistent difference that we have observed for a number of devices with four or more atomic layers of h-BN was the absolute value of the tunneling conductance through the barrier, $\sigma^{\mathrm{T}}$, which could vary by a factor of 100 for nominally the same $d$. Although this can be attributed to possible errors in determining the number of layers in thicker h-BN, ${ }^{9}$ a careful analysis of the devices' response to bias and gate voltages reveals that the reason for these variations is more likely to be inhomogeneous thickness of h-BN. We believe that in some devices one or two layers can be missing locally (in submicron-scale patches) so that the tunnel current then concentrates within these thinner areas. The cleavage of graphite is known to leave occasional stripes of smaller thickness for few-layer graphene crystals and, whereas it is possible to see missing graphene patches in an optical microscope, h-BN does not allow the required optical resolution. ${ }^{9}$

\section{CONCLUSIONS AND OUTLOOK}

We have examined the electronic properties of a new type of field-effect tunnel transistor based on graphene. This structure is an exemplar of a new class of functional materials that exploit the structural and electronic properties of graphene and related atomic layer crystals such as hexagonal boron nitride. This type of heterostructure has atomically sharp and continuous interfaces, a feature which is not presently achievable with conventional heterostructure epitaxy methods due to atomic diffusion across the interfaces. Our proposed technology provides the means of constructing, "Lego-style," a wide range of three-dimensional crystalline structures by stacking single atomic planes, layer-by-layer, in any desired sequence to achieve the required set of physical properties. We have shown that the measured gate voltage-dependent current-voltage characteristics of the transistor can be understood in terms of a simple model, which describes the device electrostatics and the wavefunction of the electrons tunneling through the boron nitride barrier. Our results point to the possibility of developing other novel devices based on gatecontrolled carrier tunneling between two graphene electrodes. For example, it should be possible to achieve gate-controlled resonant tunneling in this type of structure. ${ }^{20}$

Since the publication of Ref. 1, Yang et al. ${ }^{21}$ investigated a transistor device in which a graphene layer was placed on a layer of hydrogenated silicon. The flow of
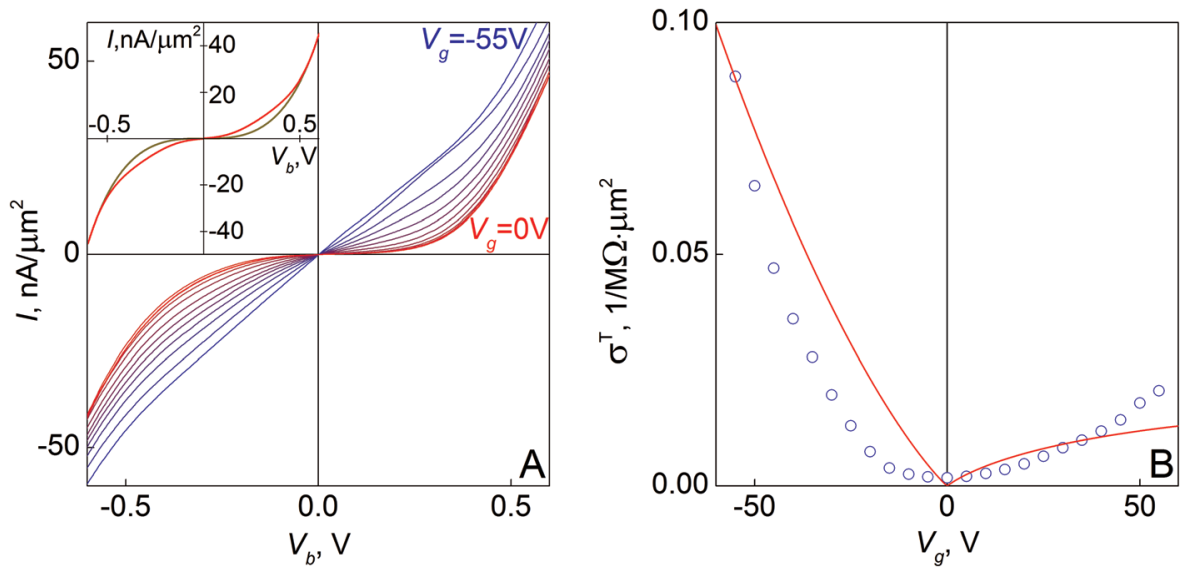

FIG. 4. h-BN/graphene/h-BN/graphene/h-BN field-effect device. (A) Tunnelling I-V curves and their response to gate voltage (in $5 \mathrm{~V}$ steps, cf. Fig. 3 of Ref. 1). The inset compares the experimental I-V at zero gate voltage (red curve) with theory (dark) which takes into account the linear density of states in the two graphene layers and assumes no momentum conservation. Temperature: $300 \mathrm{~K}$. (B) Changes in low-bias tunnelling conductance (symbols) and the theory fit for $4 \mathrm{~h}$-BN layers (solid curve). The main difference with respect to the device in Ref. 1 is a weak response at low gate voltages, which is probably due to stronger disorder and chemical doping that smears the gate influence. The electron-hole asymmetry again implies the hole tunnelling as discussed in Ref. 1. 
carriers over the Schottky barrier at the graphene/silicon interface was controlled by the gate voltage applied to a metallic electrode placed above the graphene layer. Recently, using a few atomic layer-thick crystals of semiconducting tungsten disulfide $\left(\mathrm{WS}_{2}\right)$ as the barrier material between the graphene electrodes, Georgiou et $a .^{22}$ achieved on/off switching ratios in excess of $1 \times 10^{6}$ at room temperature and demonstrated that this type of transistor structure can operate on transparent and flexible substrates.

Our article is based in part on the material presented in the supplementary online information of Ref. 1. We believe that the material should be presented separately in an archival journal to avoid our results going unnoticed by other researchers in the field.

\section{ACKNOWLEDGMENTS}

The authors gratefully acknowledge financial support from the Royal Society, Körber Foundation, and ERC.

${ }^{1}$ L. Britnell, R. V. Gorbachev, R. Jalil, B. D. Belle, F. Schedin, A. Mishchenko, T. Georgiou, M. I. Katsnelson, L. Eaves, S. V. Morozov, N. M. R. Peres, J. Leist, A. K. Geim, K. S. Novoselov, and L. A. Ponomarenko, Science 335, 947 (2012).

${ }^{2}$ L. Britnell, R. V. Gorbechev, R. Jalil, B. D. Belle, F. Schedin, M. I. Katsnelson, L. Eaves, S. V. Morozov, A. S. Mayorov, N. M. R. Peres, A. H. Castro Neto, J. Leist, A. K. Geim, L. A. Ponomarenko, and K. S. Novoselov, Nano Lett. 12, 1707 (2012).

${ }^{3}$ M. Heiblum and M. V. Fischetti, IBM J. Res. Dev. 34, 530 (1990).

${ }^{4}$ J. A. Simmons, M. A. Blount, J. S. Moon, S. K. Lyo, W. E. Baca, J. R. Wendt, J. L. Reno, and M. J. Hafich, J. Appl. Phys. 84, 5626 (1998).
${ }^{5}$ A. Zaslavsky, C. Aydin, S. Luryi, S. Cristoloveanu, D. Mariolle, D. Fraboulet, and S. Deleonibus, Appl. Phys. Lett. 83, 1653 (2003).

${ }^{6}$ A. Sciambi, M. Pelliccione, M. P. Lilly, S. R. Bank, A. C. Gossard, L. N. Pfeiffer, K. W. West, and D. Goldhaber-Gordon, Phys. Rev. B 84, 085301 (2011).

${ }^{7}$ L. Eaves, M. L. Leadbeater, D. G. Hayes, E. S. Alves, F. W. Sheard, G. A. Toombs, P. E. Simmonds, M. S. Skolnick, M. Henini, and O. H. Hughes, Solid-State Electron. 32, 1101 (1989).

${ }^{8}$ S. Luryi, Appl. Phys. Lett. 52, 501 (1988).

${ }^{9}$ R. V. Gorbachev, I. Riaz, R. R. Nair, R. Jalil, L. Britnell, B. D. Belle, E. W. Hill, K. S. Novoselov, K. Watanabe, T. Taniguchi, A. K. Geim, and P. Blake, Small 7, 465 (2011).

${ }^{10}$ L. A. Ponomarenko, R. Yang, R. V. Gorbachev, P. Blake, A. S. Mayorov, K. S. Novoselov, M. I. Katsnelson, and A. K. Geim, Phys. Rev. Lett. 105, 136801 (2010).

${ }^{11}$ M. Bokdam, P. A. Khomyakov, G. Brocks, Z. Zhong, and P. J. Kelly, Nano Lett. 11, 4631 (2011).

${ }^{12}$ R. M. Feenstra, D. Jena, and G. Gu, J. Appl. Phys. 111, 043711 (2012).

${ }^{13}$ B. Sachs, T. O. Wehling, M. I. Katsnelson, and A. I. Lichtenstein, Phys. Rev. B 84, 195414 (2011).

${ }^{14}$ J. G. Simmons, J. Appl. Phys. 34, 1793 (1963).

${ }^{15}$ E. L. Wolf, Principles of Electron Tunneling Spectroscopy (Oxford University Press, 1985).

${ }^{16}$ L. D. Landau and E. M. Lifshitz, Quantum Mechanics (Pergamon Press, Oxford, 1977).

${ }^{17}$ R. M. Ribeiro and N. M. R. Peres, Phys. Rev. B 83, 235312 (2011).

${ }^{18}$ N. Kharche and S. K. Nayak, Nano Lett. 11, 5274 (2011).

${ }^{19}$ Y. N. Xu and W. Y. Ching, Phys. Rev. B 44, 7787 (1991).

${ }^{20}$ L. Britnell, R. V. Gorbachev, A. K. Geim, L. A. Ponomarenko, A. Mishchenko, M. T. Greenaway, T. M. Fromhold, K. S. Novoselov, and L. Eaves, is "Resonant tunnelling and negative differential conductance in graphene transistors," Nature Communications (to be published).

${ }^{21}$ H. Yang, J. Heo, H. J. Song, D. H. Seo, K.-E. Byun, P. Kim, I. Yoo, H.-J. Chung, and K. Kim, Science 336, 1140 (2012).

${ }^{22}$ T. Georgiou, R. Jalil, B. D. Belle, L. Britnell, R. V. Gorbachev, S. V. Morozov, Y.-J. Kim, A. Gholinia, S. J. Haigh, O. Makarovsky, L. Eaves, L. A. Ponomarenko, A. K. Geim, K. S. Novoselov, and A. Mishchenko, Nat. Nanotechnol. 8, 100 (2013). 